\title{
Keine gegenseitige Anerkennung ohne Harmonisierung des nationalen Strafrechts in der EU
}

\author{
Sebastian Weber*
}

Die Europäische Union befindet sich in einer tiefen Krise. Dieser Satz entwickelt sich gegenwärtig zu einer Standardbeschreibung in Politik und Medien. Die Ablehnung des Verfassungsvertrages in Frankreich und den Niederlanden wie auch die Verhandlungen über den Haushalt der Union für die Jahre 2007 bis 2013 haben den Ruf der europäischen Institutionen erschüttert. Dabei hat sich die Europäische Union ehrgeizige Ziele gesetzt: Ein Raum der Freiheit, der Sicherheit und des Rechts soll Europa nach Maßgabe der Art. 2 und 29 Abs. I EUV sein. Wie anfällig aber die europäischen Zivilgesellschaften sind, haben im Juli 2005 die terroristischen Verbrechen in London auf grausame Weise wieder gezeigt. Die polizeiliche und justizielle Zusammenarbeit in Strafsachen, die so genannte Dritte Säule der Europäischen Union, ist daher zu Recht umfassend angelegt und zielt sowohl auf Präventivmaßnahmen als auch auf repressive Kriminalitätsbekämpfung ab. Im Bereich der Strafverfolgung setzt der Rat der Europäischen Union dabei vor allem das Prinzip der gegenseitigen Anerkennung nationaler Strafgesetze und Rechtsakte. Dagegen bleibt eine Harmonisierung des Strafrechts gem. Art. 29 Abs. II EUV auf das erforderliche Maß begrenzt, soll also nur dann erfolgen, wenn eine verbesserte Zusammenarbeit anders nicht zu erreichen ist. Daran, dass die unterschiedlichen nationalen Strafrechtsordnungen möglichst unberührt bleiben sollen, wird deutlich, dass die Bewahrung nationaler Souveränität und der Identität der Mitgliedstaaten das Leitbild eines noch im Werden befindlichen europäischen Strafrechts- und vor allem Strafverfolgungsraumes ist. Die Berechenbarkeit dieser Art kooperativer Strafverfolgung und letztlich damit die Rechtssicherheit des Bürgers bleiben dabei aber auf der Strecke, wie auch das Urteil des Bundesverfassungsgerichts vom 18.07.2005 gezeigt hat, durch das die Übergabe eines des Terrorismus und der Beteiligung an einer kriminellen Vereinigung verdächtigen deutschen Staatsangehörigen an Spanien gestoppt wurde.

\section{Der Europäische Haftbefehl}

Der dem Übergabeersuchen Spaniens zu Grunde liegende Europäische Haftbefehl ist gegenwärtig die prominenteste Maßnahme zur Umsetzung des Prinzips der gegenseitigen Anerkennung nationaler Strafgesetze und Rechtsakte. Durch den Rahmenbeschluss über den Europäischen Haftbefehl und die Übergabeverfahren zwischen den Mitgliedstaaten vom 13. Juni $2002(2002 / 584 / J I)^{1}$ hat das Auslieferungsrecht innerhalb der Europäischen Union beachtliche Veränderungen erfahren. Der Europäische Haftbefehl wird in Art. 1 des Rahmenbeschlusses als justizielle Entscheidung definiert, die in einem Mitgliedstaat ergeht und die Festnahme und Übergabe einer Person durch einen anderen Mitgliedstaat bezweckt. Der Ausstellungsstaat des Haftbefehls wird so in die Lage versetzt, seine Strafverfolgung, die Vollstreckung einer Freiheitsstrafe oder auch einer freiheitsentziehenden Maßnahme der Sicherung unionsweit durchzusetzen. Das System des Europäischen Haftbefehls ersetzt eine

* Sebastian Weber, Wissenschaftlicher Mitarbeiter am Institut für Internationale Angelegenheiten der Rechtswissenschaftlichen Fakultät der Universität Hamburg.

1 Amtsblatt der Europäischen Gemeinschaften (ABl. EG) 2002 Nr. L 190, 1. 
ganze Reihe völkerrechtlicher Abkommen, die bislang das Auslieferungsrecht zwischen den Mitgliedstaaten regelten, deren Komplexität und Verzögerungsrisiken jedoch für eine

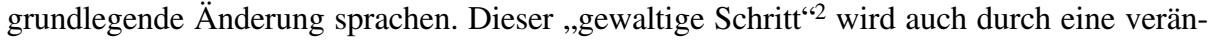
derte Terminologie unterstrichen, nach der das im klassischen Auslieferungsrecht gebräuchliche Begriffspaar „ersuchender Staat“ und „ersuchter Staat“ durch „Ausstellungsstaat“ und „Vollstreckungsstaat“ ersetzt wird, zwischen denen nunmehr eine „Übergabe gesuchter Personen“ und keine „Auslieferung“ mehr erfolgt. Das Übergabeverfahren läuft dabei ausschließlich zwischen den beteiligten Justizbehörden ab. Die das herkömmliche Auslieferungsrecht kennzeichnende abschließende, politisch geprägte Bewilligung des Ersuchens durch die jeweilige Regierung wurde damit innerhalb der Europäischen Union abgeschafft. ${ }^{3}$ Die bis dahin gerichtlich nicht kontrollierbare Entscheidung der Regierung wird so in ein justizförmiges Verfahren überführt, das allerdings auch weiterhin der freien Entscheidung der Mitgliedstaaten unterliegt, Rechtshilfe zu leisten, und wodurch auch nicht der zwischenstaatliche Charakter einer Übergabe negiert wird. ${ }^{4}$

Die materiellen Grundsätze des klassischen Auslieferungsrecht werden auch im Recht des Europäischen Haftbefehls beibehalten. So kann eine Übergabe abgelehnt werden, wenn die der gesuchten Person zur Last gelegte Handlung im Recht des Vollstreckungsstaates keine Straftat darstellt (Art. 2 IV des Rahmenbeschlusses). Das in Art. 27 II des Rahmenbeschlusses verankerte Spezialitätsprinzip ermöglicht eine Begrenzung der Strafverfolgung auf die Taten, deretwegen die Übergabe erfolgte. Der Grundsatz ne bis in idem schließt innerhalb der Europäischen Union auch weiterhin die doppelte Bestrafung einer Person aus. Darüber hinaus eröffnet der Rahmenbeschluss den Mitgliedstaaten die Möglichkeit, zahlreiche weitere obligatorische und fakultative Ablehnungsgründe einer Übergabe in die nationalen Rechtsvorschriften aufzunehmen, die zur Umsetzung des Rahmenbeschlusses erforderlich sind, damit dessen Vorgaben in den Mitgliedstaaten zu unmittelbar geltendem Recht werden. Die durch den Rahmenbeschluss bewirkten Veränderungen sind insoweit deutlich weniger einschneidend ausgefallen als zunächst geplant. So konnte sich die Europäische Kommission mit ihrem Vorschlag nicht durchsetzen, auf das Spezialitätsprinzip gänzlich zu verzichten. Den Mitgliedstaaten steht es allerdings nach Maßgabe des Art. 27 frei, weitere Erleichterungen durch entsprechende nationale Regelungen und Erklärungen einzuführen. Auch der Versuch, den Grundsatz beiderseitiger Strafbarkeit aufzugeben, scheiterte. ${ }^{5}$

Besondere Aufmerksamkeit verdient daher der Katalog des Art. 2 II des Rahmenbeschlusses, der 32 Delikte und Kriminalitätsfelder enthält, für die das Prinzip beiderseitiger Strafbarkeit nicht mehr gilt. Ist eine nach nationalem Recht eines Mitgliedstaates strafbare Handlung dieses Katalogs Gegenstand eines Europäischen Haftbefehls, so erfolgt die Übergabe dieser Person ohne Prüfung der beiderseitigen Strafbarkeit. Dieser Katalog umfasst neben Terrorismus, Menschenhandel, Kinderpornografie, klassischen Delikten wie Geiselnahme, Vergewaltigung und Tötungsdelikten, auch recht unbestimmte Begriffe wie Umweltkriminalität, Cyberkriminalität, Betrugsdelikte oder Sabotage. Voraussetzung für die Übergabe ist zwar, dass die Tat im Höchstmaß mit mindestens drei Jahren Freiheitsstrafe im Ausstellungsstaat bedroht ist, doch dürfte dies bei der deutlich überwiegenden Anzahl der Delikte und Kriminalitätsfelder der Fall sein. Der Europäische Haftbefehl führt in die-

\footnotetext{
So Helmut Satzger, in: Rudolf Streinz: EUV/EGV-Kommentar, 2003, Art. 31 EUV, Rn. 8.

Vgl. Daniel Rohlff: Der Europäische Haftbefehl (Diss. Regensburg), 2003, S. 42.

BVerfG, Urteil vom 18.07.2005 (Az.: 2 BvR 2236/04), Rz. 88.

Vgl. Wolff von Heintschel-Heinegg/Daniel Rohlff: Der Europäische Haftbefehl, GA 2003, S. 44, 49; Jörg Monar: Die EU und die Herausforderung des internationalen Terrorismus, in: Werner Weidenfeld (Hrsg.): Herausforderung Terrorismus, 2004, S. 136, 151.
} 
sem Bereich also zu einer deutlichen Vereinfachung des Übergabeverfahrens zwischen den Mitgliedstaaten, zumal innerhalb der Europäischen Union auch die Übergabe eigener Staatsangehöriger regelmäßig erfolgen soll, wie sich aus Art. 5 III des Rahmenbeschlusses ergibt. Die weltweit verbreitete Praxis, eigene Staatsangehörige zumindest nicht gegen ihren Willen einer fremden Staatsmacht zu übergeben, findet insoweit keine Anwendung mehr.

\section{Gegenseitige Anerkennung nationaler Strafgesetze und Rechtsakte als Strategie}

Der Rahmenbeschluss über den Europäischen Haftbefehl ist Teil eines Maßnahmenpaktes zur Umsetzung des Prinzips gegenseitiger Anerkennung im Strafrecht. Das Prinzip stammt ursprünglich aus dem Recht des Binnenmarktes und dient insbesondere dem Abbau von Handelshemmnissen nicht-tarifärer Art, die dem freien Verkehr von Waren, Personen und Dienstleistungen widersprechen. Durch die Übertragung dieses Prinzips auf die Strafverfolgung soll das System klassisch-völkerrechtlicher Kooperation zwischen den Mitgliedstaaten in ein System des freien Verkehrs strafrechtlicher justitieller Entscheidungen umgewandelt werden, wie der Rat der Europäischen Union in Erwägungsgrund (5) des Rahmenbeschlusses formuliert hat. Weitere Schritte zur Umsetzung dieser Strategie sind der Rahmenbeschluss über die Vollstreckung von Entscheidungen über die Sicherstellung von Vermögensgegenständen oder Beweismitteln in der Europäischen Union (2003/577/JI) vom 22. Juli $2003^{6}$, der Rahmenbeschluss über die gegenseitige Anerkennung von Geldstrafen und Geldbußen (2005/214/JI) vom 24.02.20057 und der vorgeschlagene Rahmenbeschluss über die Europäische Beweisanordnung zur Erlangung von Sachen, Schriftstücken und Daten zur Verwendung in Strafverfahren. ${ }^{8}$ Die grundlegenden Prinzipien dieser Rahmenbeschlüsse entsprechen denen, die bereits am Beispiel des Europäischen Haftbefehls dargelegt wurden. Dem Vorwurf, Europa verliere durch die getroffenen Maßnahmen seinen Charakter als liberaler Rechtsraum, versucht der Rat der Europäischen Union dadurch zu begegnen, dass er Diskriminierungsverbote ausdrücklich betont. Beispielsweise in Erwägungsgrund (6) des Rahmenbeschlusses über Sicherstellungsentscheidungen sieht der Rat eine Interpretation seiner Regelungen vor, nach der die Vollstreckung einer Sicherstellungsentscheidung verweigert werden kann, wenn diese zum Zwecke der Strafverfolgung oder Bestrafung einer Person aus Gründen ihres Geschlechts, ihrer Rasse, Religion, ethnischen Herkunft, Staatsangehörigkeit, Sprache, politischen Überzeugungen oder sexuellen Ausrichtung erlassen wurde oder die Stellung der betroffenen Person aus einem der genannten Gründe beeinträchtigt werden könnte.

Eine Ausweitung der Zusammenarbeit enthält dagegen der Rahmenbeschluss über die gegenseitige Anerkennung von Geldstrafen und Geldbußen. Der Katalog von Straftaten und Kriminalitätsfeldern, der sich hier in Art. 16 II wiederfindet, wurde um sieben Delikte beziehungsweise Deliktsgruppen ergänzt. Neben Diebstahl und Sachbeschädigung wurden die binnenmarktspezifischen Bereiche Warenschmuggel und Verletzung von Rechten an geistigem Eigentum sowie Verstöße gegen Straßenverkehrsvorschriften, insbesondere bei Lenk- und Ruhezeiten sowie beim Gefahrguttransport, und Gewalttaten gegen Personen und Bedrohung, insbesondere bei Sportveranstaltungen, aufgenommen. Schließlich wird im letzten Spiegelstrich dieses Katalogs die Entwicklungsoffenheit dieser Form der Zusammenarbeit dokumentiert. Danach erfolgt die gegenseitige Anerkennung von Geldbußen und Geldstrafen ohne Prüfung beiderseitiger Strafbarkeit auch bei Straftatbeständen, ,die vom Entscheidungsstaat

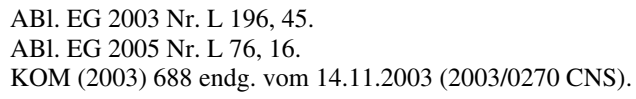


festgelegt werden und durch Verpflichtungen abgedeckt sind, die sich aus Rechtsakten der Europäischen Gemeinschaft oder aus Rechtsakten [der polizeilichen und justiziellen Zusammenarbeit in Strafsachen] nach Maßgabe des EUV ergeben“. Diese Erweiterung des Katalogs findet sich auch im Vorschlag der Kommission über die Beweisanordnung. Dadurch wird eine Verknüpfung kooperativer Strafverfolgung und -vollstreckung mit weiteren Schritten der Europäischen Union zur Harmonisierung nationaler Rechtsvorschriften hergestellt, die gerade auch das materielle Strafrecht betreffen können, wie noch zu zeigen sein wird.

\section{Rechtsstaatliche Begrenzungen des freien Übergabeverkehrs}

Ein Paradigmenwechsel ist aus deutscher Sicht die Übergabe eigener Staatsangehöriger. Ausgelöst wurde die entsprechende Grundgesetzänderung im Jahre 2000 durch das Übereinkommen über die Auslieferung zwischen den Mitgliedstaaten der EU von 1996 ${ }^{9}$, das bereits einen unbeschränkten Auslieferungsverkehr vorsah, und die Einrichtung der UN-Straftribunale für das ehemalige Jugoslawien und Ruanda sowie die damals noch bevorstehende Errichtung des Ständigen Internationalen Strafgerichtshofes. Art. 16 II GG enthielt in seiner alten Fassung ein absolutes Auslieferungsverbot für deutsche Staatsangehörige. Nach seiner Änderung erlaubt Art. 16 II 2 GG nunmehr gesetzliche Regelungen, die eine Auslieferung Deutscher an Mitgliedstaaten der Europäischen Union und Internationale Gerichtshöfe vorsehen. Ein völlig bedingungsloser Übergabeverkehr ist jedoch auf Grund der Schutz- und Fürsorgepflichten, die sich aus dem gegenseitigen Rechtsverhältnis des Staates und seiner Angehörigen ergeben, ausgeschlossen. Deutschland ist deshalb dazu verpflichtet, Eingriffe einer fremden Staatsmacht in Grundrechte eigener Staatsangehöriger abzuwehren, zumindest insoweit der Menschenwürdegehalt eines Grundrechts betroffen ist. ${ }^{10}$ Daher erlaubt Art. 16 Abs. II 2 GG die Übergabe Deutscher nur dann, wenn rechtsstaatliche Grundsätze dabei gewahrt bleiben.

Die Verweigerung einer Übergabe kommt allerdings erst dann in Betracht, wenn der betroffene Staat grundsätzlich keinen dem Grundgesetz und der Europäischen Menschenrechtskonvention im Wesentlichen vergleichbaren Grundrechtsschutz (mehr) gewährleistet. ${ }^{11}$ In diesem unwahrscheinlichen Fall würden jedoch gegen einen EU-Mitgliedstaat Maßnahmen nach Art. 7 EUV ergriffen, der die Suspendierung der Rechte des betroffenen Mitgliedstaates erlaubt, so dass auch die Kooperation bei der Strafverfolgung mit diesem Staat eingestellt werden würde. Das Bundesverfassungsgericht sieht den deutschen Gesetzgeber darüber hinaus in der Pflicht, auch unabhängig von den Mechanismen des Art. 7 EUV bei nachhaltiger Erschütterung des Vertrauens in die Rechtsstaatlichkeit der allgemeinen Verfahrensbedingungen eines Mitgliedstaates zu reagieren. ${ }^{12}$ Das in Art. 7 EUV vorgesehene Verfahren, das durch die Vertragsrevision von Nizza noch um ein Frühwarnsystem ergänzt wurde ${ }^{13}$, spricht angesichts seiner Funktion und detaillierten Regelung aber gegen unilaterale Maßnahmen einzelner Mitgliedstaaten. Einzelne durchaus erhebliche Unterschiede im nationalen Strafprozessrecht vermögen einen grundsätzlichen Einwand gegenüber dem System des Europäischen Haftbefehls daher nicht zu begründen. ${ }^{14}$ Aus rechtspolitischer

9 AB1. EG 1996 Nr. C 313, 11.

10 BVerfG, Urteil vom 18.07.2005 (Az.: 2 BvR 2236/04), Rz. 63 ff, 76.

11 Rohlff, Der Europäische Haftbefehl, 2003, S. 75; Andreas Zimmermann: Die Auslieferung Deutscher an Staaten der EU und internationale Gerichtshöfe, JZ 2001, S. 233, 237.

12 BVerfG, Urteil vom 18.07.2005 (Az.: 2 BvR 2236/04), Rz. 78.

13 Vgl. Rudolf Geiger, EUV/EGV-Kommentar, 4. Auflage 2004, Art. 7 EUV, Rn. 1.

14 Vgl. aber zu durchaus bedenklichen Beispielen Jörg Monar: EU-Antiterrormaßnahmen und die EMRK: Probleme der Kompatibilität, in: Constance Grewe/Christoph Gusy, Menschenrechte in der Bewährung, 2005, S. 223, 230; Ingo von Münch: Staatsrecht II, 5. Auflage 2002, Rn. 534d. 
Sicht erscheint seine Einführung sogar geboten, werden durch den Europäischen Haftbefehl doch auch die eigenen Strafverfolgungsinteressen gewahrt, die zweifelsohne in einem Raum offener Grenzen und des freien Verkehrs von Waren, Personen und Dienstleistungen gegenüber Angehörigen anderer Mitgliedstaaten entstehen werden. ${ }^{15}$

\section{Die Verfassungswidrigkeit der deutschen Umsetzung des Europäischen Haftbefehls}

Das Bundesverfassungsgericht wendet sich in seinem Urteil über die Verfassungsbeschwerde eines deutschen Staatsangehörigen denn auch nicht prinzipiell gegen das durch den Rahmenbeschluss vorgesehene Übergabeverfahren zwischen den Mitgliedstaaten und seine Grundsätze. Die Verfassungswidrigkeit des deutschen Umsetzungsgesetzes ergibt sich vielmehr aus Verstößen gegen verfassungsrechtliche Vorgaben, die bei der Umsetzung und insbesondere bei der Ausnutzung der nationalen Gestaltungsspielräume zu beachten gewesen wären. ${ }^{16}$

So reicht die generelle Bindung der Staatsgewalt an die Einhaltung rechtsstaatlicher Grundsätze durch Art. 16 II 2 GG für die Gewährleistung der grundrechtlich geschützten Belange eines Betroffenen nicht aus. Der Gesetzgeber ist vielmehr auf Grund des qualifizierten Gesetzesvorbehaltes verpflichtet gewesen, die Bedingungen einer Übergabe an andere Mitgliedstaaten tatbestandlich differenziert zu definieren und dabei einen die Grundrechte deutscher Staatsangehöriger möglichst schonenden Weg zu wählen. Der besondere Schutzgehalt des grundsätzlichen Auslieferungsverbots und der Verhältnismäßigkeitsgrundsatz verpflichten den Gesetzgeber insbesondere zu einer entsprechenden Ausgestaltung der Ablehnungsgründe einer Übergabe im einfachen Recht. Danach wäre gesetzlich vorzuschreiben gewesen, dass ein Deutscher auf Grund einer im eigenen Rechtsraum begangenen Tat, also einer Tat mit maßgeblichem Inlandsbezug, nicht an einen anderen Mitgliedstaat übergeben werden darf. Für Distanzdelikte, bei denen die Handlung in Deutschland, der Erfolg aber in einem anderen Mitgliedstaat eintritt, wäre eine Abwägung zwischen den Erfordernissen effektiver Strafverfolgung, der Schwere des Tatvorwurfs und den grundrechtlich geschützten Interessen des Betroffenen explizit vorzusehen gewesen. Dagegen ist eine Übergabe bei Taten mit maßgeblichem Auslandsbezug, bei denen die Handlung überwiegend in einem anderen Staat begangen wurde und der Erfolg dort eingetreten ist, unbedenklich. ${ }^{17}$ Dies gilt insbesondere bei typischerweise grenzüberschreitenden schweren Delikten, wie internationalem Terrorismus oder organisiertem Drogen- oder Menschenhandel. Die Staatsangehörigkeit schützt den Täter insoweit vor einer Übergabe nicht in gleichem Umfang, vor allem ist so eine erfolgreiche Flucht in den Heimatstaat nicht von ausschlaggebender Bedeutung.

Ferner erfordert die grundsätzliche Auslieferungsfreiheit des Art. 16 II 1 GG für deutsche Staatsangehörige weitere Schutzklauseln. Der Gesetzgeber muss nach dem Urteil des Bundesverfassungsgerichts unter anderem dafür Sorge tragen, dass die im Rahmenbeschluss vorgesehenen möglichen Bedingungen für eine Übergabe, etwa die Rücküberstellung zur Vollstreckung der Strafe (Art. 5 III), auch tatsächlich eingehalten werden können. Es seien zudem Regelungen erforderlich für den Fall, dass ein Übergabeersuchen eigener Strafverfolgung zuvorkomme.

15 Mark Deiters: Gegenseitige Anerkennung von Strafgesetzen in Europa, ZRP 2003, S. 359, 360.

16 BVerfG, Urteil vom 18.07.2005 (Az.: 2 BvR 2236/04), Rz. 84 ff.

17 Anderer Ansicht allerdings Richter Broß, der in seiner abweichenden Meinung grundsätzliche Zweifel an der Vereinbarkeit des Rahmenbeschlusses mit dem Subsidiaritätsprinzip formuliert, Rz. $132 \mathrm{ff}$. 
Gegen die Rechtsschutzgarantie des Art. 19 IV GG verstößt zudem der Ausschluss der Anfechtbarkeit der Bewilligungsentscheidung einer Übergabe durch § 74b des Gesetzes über internationale Rechtshilfe (IRG), da die abschließende Aufzählung von fünf Sachverhaltskonstellationen in $\S 83 \mathrm{~b}$ IRG, die zur Ablehnung einer Übergabe führen können, den Charakter der Bewilligungsentscheidung maßgeblich verändert hat. Während vormals nur unbenannte, gerichtlich nicht überprüfbare außen- und allgemeinpolitische Erwägungen für die politische Bewilligungsentscheidung ausschlaggebend waren, sei nunmehr ein verrechtlichter Abwägungsprozess vorgesehen, an dessen Ende eine zu begründende Ermessensentscheidung der zuständigen Behörde steht, die der Überprüfung durch die rechtsprechende Gewalt unterliegen müsse.

Kommt der Gesetzgeber diesen Bedingungen bei der Neufassung des Umsetzungsgesetzes des Rahmenbeschlusses jedoch nach, scheidet eine Verletzung des Rückwirkungsverbots des Art. 103 II GG von vornherein aus. Allerdings könnte ein Verstoß vorliegen, wenn ein bislang vor Auslieferung absolut geschützter Deutscher für Handlungen, die keinen maßgeblichen Auslandsbezug aufwiesen und zum Zeitpunkt der Tat in Deutschland straffrei gewesen sind, sich nunmehr in einem anderen Mitgliedstaat verantworten müsse. Obwohl das Rückwirkungsverbot nicht für das Strafverfahrensrecht gelte, zu dem auch das Auslieferungsrecht zu zählen sei, käme dies nach Ansicht des Bundesverfassungsgerichts einer materiell rückwirkenden Rechtsänderung möglicherweise gleich.

\section{Die Bewahrung von Souveränität und Identität der europäischen Verfassungsstaaten}

Die von ihm selbst in der mündlichen Verhandlung über die Verfassungsbeschwerde aufgeworfene Frage, ob die Mitgliedstaaten nicht mittlerweile zu viel ihrer Staatlichkeit auf die europäische Ebene transferiert hätten ${ }^{18}$, beantwortet das Bundesverfassungsgericht dagegen zu Recht negativ. Das völkerrechtsfreundliche Grundgesetz enthalte keine unüberwindbaren Hürden für die Auslieferung eigener Staatsangehöriger, die auch nicht zu einer unzulässigen Entstaatlichung Deutschlands durch das System des Europäischen Haftbefehls führe. ${ }^{19}$

Das Strafrecht gilt allerdings nicht zu Unrecht als klassische Domäne der Nationalstaaten, drücken sich doch bestimmte Ordnungsvorstellungen eines Gemeinwesens im Strafrecht explizit aus und verdeutlicht der absolute Strafanspruch die Effektivität einer Staatsmacht. Strafgesetzgebung und Kriminalpolitik sind jedoch nicht nur durch rationale Überlegungen geprägt, sondern sie unterliegen maßgeblich auch kulturellen und historischen Besonderheiten, Wertvorstellungen und Ängsten. Deshalb ergeben sich innerhalb der Europäischen Union auch veritable Unterschiede zwischen den Mitgliedstaaten, ob und, wenn ja, mit welcher Intensität bestimmte Handlungen verfolgt und bestraft werden. ${ }^{20}$ Prinzipiell verdient dabei die Entscheidung eines Staates, eine Handlung nicht unter Strafe zu stellen oder sie als bloße Ordnungswidrigkeit zu betrachten, die gleiche Anerkennung, wie die Entscheidung eines anderen Staates, dieselbe Handlung als Straftat zu definieren. Das dergestalt geprägte Strafrecht trägt so maßgeblich zur Identität eines Staates bei, ist es doch ein handfester Ausdruck gesellschaftlicher Werturteile und bildet dadurch den Konsens einer Gesellschaft ab. Das völkerrechtliche Institut der Souveränität dient nicht zuletzt auch dem Schutz vor Eingriffen von außen in diese Entscheidungen und sichert damit die Identität eines Staatswesens.

18 Vgl. die Berichterstattung bei Reinhard Müller: Ein naheliegendes Instrument?, Frankfurter Allgemeine Zeitung vom 14.04.2005, S. 4.

19 BVerfG, Urteil vom 18.07.2005 (Az.: 2 BvR 2236/04), Rz. 70 ff.

20 Vgl. Frederik Rüter: Harmonie statt Dissonanz, ZStW 105 (1993), S. 30, 35. 
Der souveräne Staat ist zwar durch eigenständige, unabgeleitete Herrschaftsgewalt gekennzeichnet, doch steht er gleichwohl nicht über dem Völkerrecht, sondern er ist Teil der Völkerrechtsordnung, die Inhalt und Schranken seines Rechtsstatus definiert. Der freiwillige Eintritt eines Staates in ein völkerrechtliches Vertragsregime ist Ausdruck dieser Souveränität und bedeutet nicht etwa den (partiellen) Verlust derselben, wenngleich ein solcher Akt zu deutlichen Beschränkungen souveräner Machtausübung führen kann, wie das Beispiel der Europäischen Gemeinschaften zeigt. Je mehr sich die Staaten bei der Bewältigung ihrer Aufgaben internationaler Zusammenschlüsse bedienen, desto mehr entfernen sie sich allerdings vom klassischen Souveränitätsverständnis, das durch die Ausschließlichkeit der dem territorialen Souverän zugewiesenen Herrschaftsbefugnisse gekennzeichnet ist.

Es verwundert daher auch nicht, dass die Mitgliedstaaten der Europäischen Union in Art. 6 III EUV deshalb die Wahrung ihrer Identität zu einer grundlegenden Maßgabe der Europäischen Einigung gemacht haben. Rechtlich und tatsächlich bleiben aber heute immer weniger Sachfragen übrig, die ausschließlich von einzelnen Staaten entschieden werden können. Grenzüberschreitende Kriminalität und internationaler Terrorismus stellen insoweit besonders hervorzuhebende Herausforderungen dar. Einerseits schützt die staatliche Souveränität zwar Wertentscheidungen eines Staatsvolkes im Rahmen seines Verfassungsstatus, andererseits bestehen eindeutige Interessen der Staatengemeinschaft und mit Blick auf den einzelnen Bürger auch (Schutz-)Pflichten, die anstehenden politischen Probleme gemeinsam zu lösen. ${ }^{21}$

Die gegenseitige Anerkennung nationaler Strafgesetze ist dabei ein denkbarer Weg zu größerer Kooperation, die etwa durch das Instrument des Europäischen Haftbefehls operabel wird. Die nationalen Strafrechtsordnungen bleiben dabei weitgehend unberührt, vielmehr wird ihnen im Zweifel unionsweit zur Durchsetzung verholfen.

Aus der Perspektive des Bürgers erscheint der eingeschlagene Weg, dieses Ziel durch ein möglichst großes Maß gegenseitiger Anerkennung und ein möglichst geringes Maß an nivellierender Harmonisierung zu erreichen, jedoch durchaus fragwürdig. Die gegenseitige Anerkennung nationaler Strafgesetze stellt ein nationale Besonderheiten möglichst schonendes Mittel zur Verbesserung europaweiter Kriminalitätsbekämpfung dar, doch birgt es für den Bürger erhebliche (Rechts-)Unsicherheiten. Das Prinzip der gegenseitigen Anerkennung beantwortet nämlich nicht die Frage, welches nationale Strafrecht für welche Handlung letztlich maßgebend ist. Dies entscheidet sich vielmehr nach den nationalen Bestimmungen, die die Anknüpfungspunkte für die Begründung eines Strafanspruchs definieren. So zeigt sich beispielsweise das deutsche Recht beziehungsweise die Rechtsprechung besonders zuständigkeitswillig. Der Bundesgerichtshof hält etwa einen Ausländer, der auf einem ausländischen Server Inhalte deutschen Internetusern zugänglich macht, nach deutschem Recht für strafbar, wenn der zum Tatbestand gehörende Erfolg der öffentlichen Leugnung des Holocausts und der Volksverhetzung dadurch in Deutschland eintritt ( $§ 130$ I, III StGB).22 Im konkreten Fall mag dies rechtspolitisch noch erstrebenswert erscheinen, mit Blick auf den Europäischen Haftbefehl ist dieses Ergebnis aber höchst problematisch, kann der einzelne Bürger doch kaum mehr überblicken, welche Norm welchen nationalen Strafrechts er möglicherweise durch eine Handlung verletzt, obwohl er sich nach Maßgabe seines heimatlichen Strafrechts, das sein Rechtsempfinden am ehesten bestimmen dürfte, womöglich rechtstreu

21 Vgl. Stefan Oeter: Souveränität - ein überholtes Konzept?, in: Hans J. Cremer/Thomas Giegerich/Dagmar Richter/Andreas Zimmermann: Tradition und Weltoffenheit des Rechts, Festschrift Steinberg, Berlin u.a. 2002, S. 259, $276 \mathrm{ff}$.

22 BGHSt 46, 212. 
verhält. Der Europäische Haftbefehl könnte dazu führen, dass eine Person wegen einer solchen Handlung einem anderen Mitgliedstaat zum Zwecke der Strafverfolgung übergeben wird. ${ }^{23}$ Als Anknüpfungspunkte für einen nationalen Strafanspruch dienen dabei sowohl der Handlungs- wie der Erfolgsort, die Staatsangehörigkeit des Täters oder auch des Opfers. Innerhalb der Europäischen Union begegnen sich in kaum mehr überschaubarer Häufigkeit Waren, Personen und Dienstleistungen, die so gegebenenfalls zur Begründung eines nationalen Strafanspruchs herangezogen werden können. Wird diese ohnehin schon für den Einzelnen unübersichtliche Lage nun ergänzt durch den vom Rat beabsichtigten ,freien Verkehr strafrechtlicher Entscheidungen“" entsteht ein noch größeres Maß an Rechtsunsicherheit.

Das in Art. 103 II GG und Art. 7 der Europäischen Menschenrechtskonvention verankerte Gesetzlichkeitsprinzip im Strafrecht (nullum crimen, nulla poena sine lege) kann als eine der wesentlichen Ausprägungen eines Rechtsstaates angesehen werden. Als allgemeiner Rechtsgrundsatz bindet das Gesetzlichkeitsprinzip über Art. 6 II EUV auch die Europäische Union. ${ }^{24}$ Über das Rückwirkungs- und Analogieverbot hinaus ist ihm ein Bestimmtheits- und Klarheitsgebot für gesetzliche Straftatbestände zu entnehmen. ${ }^{25}$ Dieses Gebot verpflichtet zu hinreichend klar und bestimmt formulierten Gesetzen, aus denen sich die strafbare Handlung und die Strafe eindeutig ergeben. Die Entwicklung klarer Konturen eines Straftatbestandes durch richterliche Auslegung ist dadurch zwar nicht ausgeschlossen, doch muss diese mit dem Wesen des Straftatbestandes vereinbar und für den Betroffenen ausreichend vorhersehbar sein. ${ }^{26}$ Zwar wird das Gesetzlichkeitsprinzip durch den Rahmenbeschluss über den Europäischen Haftbefehl nicht verletzt, erfolgt doch die Strafgesetzgebung für die Katalogtaten des Art. 2 II des Rahmenbeschlusses weiterhin durch die nationalen Parlamente, doch dürfte die Vorhersehbarkeit einer Verurteilung für den Betroffenen angesichts der Unbestimmtheit einzelner Begriffe des Katalogs und der gegebenenfalls 25 verschiedenen Ausprägungen im nationalen Strafrecht der Mitgliedstaaten - etwa beim Betrug - recht zweifelhaft erscheinen. Der Europäische Haftbefehl führt aber im Falle einer Katalogtat unter Umständen zur Unterwerfung unter eine mitgliedstaatliche Strafrechtsordnung, die so für den Betroffenen kaum vorhersehbar gewesen sein dürfte.

In gewisser Weise stehen sich demnach die Rechtssicherheit des Bürgers und die Bewahrung der Identität der Mitgliedstaaten gegenüber. Gerecht werden muss die Union aber beiden Belangen in gleicher Weise. Das berechtigte Interesse an der Achtung ihrer Identität müssen die Mitgliedstaaten deshalb in Ausgleich bringen mit dem Interesse des Bürgers an der Vorhersehbarkeit strafbarer Handlungen und einer entsprechenden Verurteilung.

\section{Materielle Strafrechtsharmonisierung durch Rahmenbeschlüsse - eine Alternative?}

Von besonderer Bedeutung ist in der Debatte darüber hinaus die Befürchtung, durch die gegenseitige Anerkennung setze sich innerhalb der Europäischen Union die jeweils strengste Strafrechtsordnung durch. ${ }^{27}$ Dass dies im Zweifel nur bei den bereits angeführten Distanzdelikten der Fall ist, wenn also Handlungs- und Erfolgsort auseinanderfallen und dadurch mehrere nationale Strafansprüche miteinander konkurrieren, macht diesen Einwand noch nicht unbeachtlich. In Kombination mit der gesteigerten Unberechenbarkeit von Straf-

23 Vgl. zur Kritik Arnd Koch, Nationales Strafrecht und globale Internetkriminalität, GA 2002, S. $702,707$.

24 EuGH, Urteil vom 12.12.1996, verb. Rs. C-74/95 und C-129/95, Strafverfahren gegen X, Slg. 1996, I-6609.

25 Vgl. Christoph Grabenwarter: Europäische Menschenrechtskonvention, 2. Aufl., München 2005, § 24 Rn. 136.

26 EGMR, Urteil vom 22.11.1995, S.W., Nr. 20166/92, Serie A 335-C, Z. 34 ff.

27 Vgl. Bernd Schünemann: Fortschritte und Fehltritte in der Strafrechtspflege der EU, GA 2004, S. 193, 202. 
verfolgung und Verurteilung sowie den weiteren Möglichkeiten zur Begründung eines Strafanspruchs erlangt diese Befürchtung vielmehr Bedeutung.

Ein größeres Maß an Berechenbarkeit für den Einzelnen ließe sich durch die Harmonisierung materieller Straftatbestände erzielen. Der Einzelne könnte sich dann darauf verlassen, dass eine vergleichbare Strafbarkeit für Handlungen unionsweit besteht, auf Grund derer eine Übergabe an andere Mitgliedstaaten erfolgen kann. Die Harmonisierung materiellen Strafrechts sieht Art. 31 I lit. e EUV bislang aber ausweislich des Wortlauts nur für Terrorismus, organisierte Kriminalität und illegalen Drogenhandel vor. Schrifttum und Praxis gehen allerdings überwiegend davon aus, dass jedes Delikt, das besonders schwere und grenzüberschreitende Kriminalität betrifft, harmonisierungsfähig ist, wenngleich dies angesichts der Eindeutigkeit des Wortlauts durchaus zweifelhaft erscheint. ${ }^{28}$ Harmonisierung bedeutet insoweit die Festlegung von Mindestvorschriften über die Tatbestandsmerkmale und die Strafen. Eine vollkommene Rechtsvereinheitlichung lässt sich dagegen nicht auf die Vorschriften des EUV stützen. Den Mitgliedstaaten verbleibt vielmehr die Möglichkeit, über die Mindestvorgaben hinauszugehen und damit deutliche Akzente im Sinne nationaler Eigenheiten zu setzen.

Die Einwirkungsmöglichkeiten eines Rahmenbeschlusses sind dabei allerdings nicht zu unterschätzen. Der Rahmenbeschluss zur Terrorismusbekämpfung (2002/475/JI) vom 13. Juni $2002^{29}$ enthält etwa durchaus detaillierte und weitreichende Vorgaben für die nationale Strafgesetzgebung der Mitgliedstaaten. So muss etwa gem. Art. 5 III dieses Rahmenbeschlusses das Anführen einer terroristischen Vereinigung (definiert in Art. 2 II lit. a) seitens der Mitgliedstaaten mit einer Höchststrafe von mindestens 15 Jahren, die Beteiligung an den Handlungen einer terroristischen Vereinigung (definiert Art. 2 II lit. b) mit einer Höchststrafe von mindestens acht Jahren versehen werden. Durch diese Maßgaben und die Definition einer terroristischen Vereinigung in Art. 2 I wird der Handlungsspielraum der Mitgliedstaaten bei der Ausgestaltung der nationalen Rechtsordnung damit erheblich eingeschränkt. Den Mitgliedstaaten steht es allerdings frei, die Tatbestandsdefinition einer terroristischen Vereinigung weiter zu fassen und die Mindeststrafe höher anzusetzen. Strafschärfende Regelungen bleiben also möglich, den Mitgliedstaaten ist es jedoch verwehrt, hinter den Mindestvorschriften zurückzubleiben.

Der Rahmenbeschluss erweist sich so als ein durchaus wirkungsvolles Handlungsinstrument der Europäischen Union, das den Mitgliedstaaten ein Ziel verbindlich bereits durch den Ratsbeschluss vorgibt, ihnen aber Form und Mittel der Umsetzung in nationales Recht überlässt. Solange rechtsstaatliche Prinzipien wie das Rückwirkungs- und Analogieverbot von Strafgesetzen beachtet werden, ist auch die rahmenbeschlusskonforme Auslegung des nationalen Rechts geboten. ${ }^{30}$ Dem Rahmenbeschluss kommt dabei allerdings keine supranationale Vorrangwirkung zu. Die rahmenbeschlusskonforme Auslegung ist vielmehr eine Methode, bestehendes Recht an gegebenenfalls neuen Vorgaben auszurichten. Der rahmenbeschlusskonformen Auslegung kommt dabei kein absoluter Vorrang vor anderen (nationalen) Auslegungsmethoden zu, wenngleich jede rahmenbeschlusswidrige Auslegung ausscheidet und der Rahmenbeschlusskonformität jedenfalls im Falle der beabsichtigten Umgestaltung nationalen Rechts Vorrang einzuräumen ist. ${ }^{31}$ Dieser Grundsatz verlangt dem

28 Vgl. Helmut Satzger: Internationales und Europäisches Strafrecht, Baden-Baden 2005, § 8 Rn. 43; Röben, in: Eberhard Grabitz/Meinhard Hilf (Hrsg.): Das Recht der Europäischen Union - Kommentar (Loseblattsammlung, Stand: 2000), Art. 31 EUV, Rn. 13; a.A. etwa Bernd Schünemann: Ein Gespenst geht um in Europa Brüsseler ,Strafrechtspflege“ intra muros, GA 2002, S. 501, 504.

29 AB1. EG 2002 Nr. L 164, 3.

30 EuGH, Urteil vom 16.06.2005, Rs. C-105/03, Pupino, Rz. 43 f.

31 Vgl. Martin Nettesheim: Auslegung und Fortbildung nationalen Rechts im Lichte des Gemeinschaftsrechts, AöR 119 (1994), S. 261, 274 ff.; Bernd Hecker: Europäisches Strafrecht als Antwort auf transnationale Kriminalität?, JA 2002, S. 723, 728. 
nationalen Rechtsanwender auf Grund eines Rahmenbeschlusses allerdings keine Auslegung contra legem ab, wohl aber eine Berücksichtigung der gesamten Rechtsordnung, wenn dadurch eine rahmenbeschlusskonforme Auslegung der nationalen Vorschrift ermöglicht wird. ${ }^{32}$ Bedeutung erlangt diese Wirkung eines Rahmenbeschlusses gerade auch im Strafverfahrensrecht, etwa bei Aussagemodalitäten oder anderen Formen der Beweiserhebung.

Die demokratische Legitimation dieser Form gubernativer Rechtsetzung wird durchaus kritisch hinterfragt. ${ }^{33}$ Maßgebliches Organ der Rechtsetzung ist der Rat der Europäischen Union, dem die Vertreter der Regierungen der 25 Mitgliedstaaten angehören. Das Europäische Parlament besitzt dagegen gem. Art. 39 I EUV lediglich ein Anhörungsrecht. Zwar erfolgt die Umsetzung eines Rahmenbeschlusses durch Beschlussfassung der nationalen Parlamente, doch sind diese durch die Zielvorgabe des Rahmenbeschlusses gebunden, also nicht mehr frei in der Entscheidung, ob sie einen entsprechenden Rechtsakt erlassen, sondern nur noch wie die Vorgaben des Rahmenbeschlusses erfüllt werden. ${ }^{34}$ Die mittelbare demokratische Legitimation der Regierungsvertreter durch ihre nationale Wahl in das Regierungsamt hebt diesen Mangel nur bedingt auf. In diesem Zusammenhang erscheint das zumindest vorläufige Scheitern des Verfassungsvertrages bedauerlich, sieht dieser doch eine gemeinsame Rahmengesetzgebungskompetenz von Parlament und Rat bei der Strafrechtsharmonisierung vor.

Das Einstimmigkeitserfordernis dürfte jedoch eine wünschenswerte Beschränkung dieser Rechtsetzung auf das Erforderliche bewirken. Das Konsensprinzip, das im herkömmlichen Völkerrecht den Schöpfungsprozess seiner Normen legitimiert und die Ableitung des Rechts der Staatengemeinschaft von den Völkerrechtssubjekten beschreibt, unterliegt bei Rechtssetzungsprozessen in überstaatlichen Institutionen jedoch einem deutlichen Wandel. Es ist dabei zu beachten, dass eine Auflösung des Legitimationszusammenhangs zu schweren Akzeptanzproblemen in diesem Sinne dann autoritativer Rechtsetzung führen wird, wie sich möglicherweise bei den Volksabstimmungen über den Verfassungsvertrag bereits gezeigt hat. Die Konstruktion der Union geht erheblich über die ursprüngliche völkerrechtliche Basis hinaus, die Rückbindung der politischen Willensbildung an die Nationalstaaten und die sie tragenden Parlamente ist bislang allerdings weiterhin unverkennbar. Im Ergebnis (!) wird die Harmonisierung materiellen Strafrechts durch Rahmenbeschlüsse meist kaum kritikwürdig erscheinen, dürfte doch der Kompromiss zwischen 25 Mitgliedstaaten, der gerade unter Berücksichtigung der nationalen Eigenarten zu erzielen sein wird, im Zweifel ausgewogener ausfallen als manches nationale Strafgesetz, das von einer bestimmten, parteipolitisch orientierten Parlamentsmehrheit beschlossen wird. ${ }^{35}$

\section{Die Erforderlichkeit einer ausgewogenen Strategie}

Die justizielle Zusammenarbeit in Strafsachen kann durch die gegenseitige Anerkennung nationaler Strafgesetze zu einem wirkungsvollen Instrument bei der unionsweiten Kriminalitätsbekämpfung werden. Durch den Europäischen Haftbefehl, die Vollstreckung von Sicherstellungsentscheidungen wie auch die gegenseitige Anerkennung von Geldbußen und Geldstrafen wächst die Europäische Union auch im Bereich der Strafverfolgung deutlich en-

32 EuGH a.a.O., Rz. 47.

33 Vgl. hierzu grundsätzlich Armin von Bogdandy: Gubernative Rechtsetzung, Habil. FU Berlin 1996, Tübingen 2000; kritisch Klaus Lüderssen: Europäisierung des Strafrechts und gubernative Rechtssetzung, GA 2003, 71.

34 Winfried Brechmann, in: Christian Callies/Matthias Ruffert (Hrsg.): EUV/EGV-Kommentar, 2. Auflage, Neuwied u.a. 2002, Art. 34 EUV, Rn. 7; Martin Böse, in: Jürgen Schwarze (Hrsg.), EU-Kommentar, Baden-Baden 2000, Art. 34 EUV, Rn. 6.

35 So auch Joachim Vogel: Harmonisierung des Strafrechts in der Europäischen Union, GA 2003, S. 314, 332. 
ger zusammen. Ein europäischer Strafrechtsraum könnte auf diese Weise zu einer sinnvollen Antwort auf die Herausforderung des internationalen Terrorismus wie auch der organisierten Kriminalität werden. ${ }^{36}$

Ausgewogen ist diese Strategie aber bislang noch nicht. Die Furcht vor Verlust mitgliedstaatlicher Identität und Souveränität, die eine klare Präferenz zu Gunsten des Prinzips gegenseitiger Anerkennung hervorbringt, bringt die unionsweite Zusammenarbeit aus dem rechtsstaatlichen Gleichgewicht. Integrations- und bürgerfreundlich wäre vielmehr eine durch die Harmonisierung des materiellen Strafrechts wie der Verfahrensgrundsätze erzielbare Verlässlichkeit und Berechenbarkeit kooperativer Strafverfolgung. Die Effektivität der Strafverfolgung und die Rechtssicherheit des Bürgers sind gleichermaßen Prämissen einer wirkungsvollen, aber auch akzeptablen unionsweiten Zusammenarbeit. Der Subsidiaritätsgrundsatz gebietet dabei einen Weg, der genügend Raum für die Rechtstraditionen und -kulturen der Mitgliedstaaten lässt. Dass aber die gegenseitige Anerkennung der Harmonisierung deshalb grundsätzlich vorzuziehen ist, um die nationale Identität und Staatlichkeit der Mitgliedstaaten zu wahren, wie das Bundesverfassungsgericht anmerkt $\mathrm{t}^{37}$, kann dagegen nicht überzeugen. Nach Maßgabe der Kompetenzgrundlagen des EUV kann vielmehr auch in der dargelegten Form die Harmonisierung nationalen Strafrechts erfolgen, die ja gerade nicht zu einer vollständigen Rechtsvereinheitlichung führen darf. Kann das Regelungsziel, die effektivere Zusammenarbeit, sowohl durch die gegenseitige Anerkennung wie die Harmonisierung nationaler Strafgesetze erreicht werden, liegen vielmehr zwei funktionsgleiche Regelungsalternativen vor. Der Subsidiaritätsgedanke gibt insoweit nicht den Ausschlag für die gegenseitige Anerkennung als leitendes Prinzip, sondern er verlangt vielmehr, dass der Weg eingeschlagen wird, der die nationalen Rechtsordnungen am wenigsten berührt, der aber zugleich den Bürgerrechten und der Rechtsstaatlichkeit genügt, die als maßgebende Werte ebenfalls in Art. 6 EUV niedergelegt sind. ${ }^{38}$

Im Zusammenspiel mit weit gefassten nationalen Strafansprüchen besteht in Anwendung des Prinzips gegenseitiger Anerkennung unter Verzicht auf die Prüfung beiderseitiger Strafbarkeit aber die Möglichkeit, dass sich die jeweils strengere Strafrechtsordnung durchsetzt und in einzelnen Mitgliedstaaten dadurch anerkannte bürgerliche Freiheiten beschnitten werden. Man denke nur an stark umstrittene Fragen des Abtreibungsrechts, der Sterbehilfe oder auch des Betäubungsmittelrechts. Reformbedürftig erscheint deshalb vor allem das Strafanwendungsrecht der Mitgliedstaaten. Innerhalb eines Raumes der Freiheit, der Sicherheit und des Rechts bedürfte es der unionsweiten Festlegung auf ein Prinzip zur Ermittlung der jeweils anwendbaren Rechtsordnung. Die Lösung der skizzierten Problemkonstellationen sollte mit Blick auf schwere Straftaten wie Terrorismus oder Kinderpornographie dagegen nicht in einer Reduzierung kooperativer Strafverfolgung und der Aufrechterhaltung von Modellen gesucht werden, die vor allem eigene Staatsangehörige vor einer Übergabe bewahren sollen. Vorzugswürdig dürfte dagegen die unionsweite Geltung des Tatortprinzips als Anknüpfungspunkt sein, stellt doch die den Tatbestand erfüllende Handlung den entscheidenden Verstoß gegen die das gesellschaftliche Leben regelnde Strafnorm dar, wenngleich erst der zum Tatbestand gehörende Erfolg zur eigentlichen Störung des Rechtsfriedens führt. ${ }^{39}$

36 Vgl. aber die Kritik die Peter-Alexis Albrecht, Die vergessene Freiheit, 2003.

37 BVerfG, Urteil vom 18.07.2005 (Az.: 2 BvR 2236/04), Rz. 75.

38 So auch die abweichende Meinung der Richterin Lübbe-Wolff, BVerfG, Urteil vom 18.07.2005 (Az.: 2 BvR 2236/04), Rz. $160 \mathrm{f}$.

39 So auch Deiters: Gegenseitige Anerkennung von Strafgesetzen in Europa, S. 361; Rüter: Harmonie statt Dissonanz, S. 46. 
Die in den Katalogen der Rahmenbeschlüsse aufgeführten 32 beziehungsweise 39 Delikte und Deliktsgruppen markieren umfangreiche Kriminalitätsbereiche, bei denen eine unionsweite Zusammenarbeit unter Verzicht auf bisherige Beschränkungen des Rechtshilferechts erfolgen soll. Vage Begriffe wie Umwelt- oder Cyberkriminalität verlangen dabei geradezu nach Definition und Harmonisierung. Tatbestände wie Geldfälschung oder Geldwäsche sind bereits in entsprechenden Rahmenbeschlüssen angeglichen worden. Der Rahmenbeschluss über Angriffe auf Informationssysteme harmonisiert einen Teil der unter den Begriff der Cyberkriminalität zu fassenden Handlungen. ${ }^{40}$ Gegenseitige Anerkennung und Harmonisierung bedingen einander also und können nicht als vollkommene Alternativen behandelt werden. Die Wertung der Art. 29 II, 31 I lit. e EUV darf daher nicht dazu führen, der gegenseitigen Anerkennung den grundsätzlichen Vorzug einzuräumen und die Harmonisierung zu begrenzen. Die Erforderlichkeit einer Harmonisierung im Sinne dieser Vorschriften ist vielmehr dann anzunehmen, wenn die gegenseitige Anerkennung etwa mittels des Europäischen Haftbefehls zur unionsweiten Durchsetzung stark divergierender nationaler Straftatbestände führt und die Berechenbarkeit der Strafverfolgung und einer Verurteilung darunter leidet.

Die Europäische Union kann demnach mit Hilfe ihrer Instrumente zu einem effektiven Strafverfolgungsraum werden, der als Teil eines Raumes der Freiheit, der Sicherheit und des Rechts anerkennenswert ist. Klare Zuständigkeitsregelungen durch eine Reform des nationalen Strafanwendungsrechts und die Harmonisierung des materiellen Strafrechts wie der Verfahrensgrundsätze sind aber Voraussetzung für eine rechtsstaatlich akzeptable weit gefasste gegenseitige Anerkennung nationaler Strafgesetze und Rechtsakte, soll der Bürger nicht zum Spielball einer für ihn nicht mehr überschaubaren Kooperation zwischen den Strafverfolgungsbehörden der Mitgliedstaaten werden.

40 ABl. EG 2005 Nr. L 69, 67. 